\title{
A THEOREM ON THE EXTENSION OF A RECTANGULAR MATRIX OF CONTINUOUS FUNCTIONS TO BECOME A NONSINGULAR SQUARE MATRIX
}

\author{
H. F. MATHIS
}

TheOREM. If the elements $a_{i j}(t)(i=1, \cdots, p<q ; j=1, \cdots, q)$ of $a p \times q$ rectangular matrix $A$ are continuous functions of $n$ variables ${ }^{1}$ $t_{1}, \cdots, t_{n}$ on a closed region $R$ which is homeomorphic to a closed $n$-cell and $A$ has maximum rank everywhere on $R$, then there exist polynomials $b_{k j}(t)(k=p+1, \cdots, q)$ in $t$ such that the matrix

$$
M=\left(\begin{array}{l}
a_{i j}(t) \\
b_{k j}(t)
\end{array}\right)
$$

is nonsingular everywhere on the closed region $R$.

There exists a homeomorphic mapping $u=\phi(t)\left(u=u_{1}, \cdots, u_{n}\right)$ of $R$ onto a closed $n$-dimensional cube $K$ with one vertex at the origin and with the $n$ coordinate axes as edges. The matrix $B=\left(a_{i j}\left(\phi^{-1}(u)\right)\right.$ $\left.=\alpha_{i j}(u)\right)$ has maximum rank everywhere on $K$ and its elements are continuous on $K$. If the functions $\beta_{1}(u), \cdots, \beta_{q}(u)$ are continuous solutions of the equations

$$
\sum_{j=1}^{q} \alpha_{i j}(u) \beta_{j}(u)=0 \quad(i=1, \cdots, p)
$$

which do not vanish simultaneously on $K$, the matrix

$$
C=\left(\begin{array}{c}
\alpha_{i j}(u) \\
\beta_{j}(u)
\end{array}\right)
$$

has maximum rank everywhere on $K$.

At any point $\bar{u}$ on $K$ at least one of the $p \times p$ determinants of the matrix $B$ is different from zero in a neighborhood of $\bar{u}$. Consequently the cube $K$ can be divided into a finite number of cubes $k_{\delta}$, the length of whose edges are equal and whose edges are parallel to the $n$ coordinate axes, so that for each cube $k_{\delta}$ at least one $p \times p$ determinant of the matrix $B$ does not vanish on the closed cube $k_{\delta}$. If the proper $q-p \beta$ 's are arbitrarily chosen on one of the cubes $k_{\delta}$ so that they are continuous, the remaining $\beta$ 's will be uniquely determined and con-

Received by the editors March 15, 1949.

1 For the special case where $n=1$, see G. A. Bliss The problem of Mayer with variable end-points, Trans. Amer. Math. Soc. vol. 19 (1918) p. 312. 
tinuous on $k_{\delta}$. At any point $\bar{u}$ on the cube $K$, more than one $p \times p$ determinant may be different from zero and corresponding to each of these determinants there is a different set of arbitrary $\beta$ 's. If any one of these sets of arbitrary $\beta$ 's vanish simultaneously at $\bar{u}$, all of the $\beta$ 's must vanish at $\bar{u}$.

Starting with the small cube which has a vertex at the origin, denote this closed cube by $k_{1}$ and choose the arbitrary $\beta$ 's for this cube so that they are continuous and do not vanish simultaneously on $k_{1}$. Next choose any cube $k_{2}$ which has a common face with $k_{1}$. By the preceding argument, the arbitrary $\beta$ 's for $k_{2}$ can be extended as constants along straight lines through the origin. When the arbitrary $\beta$ 's for $k_{2}$ have been extended in this manner, the $\beta$ 's are uniquely determined, continuous, and do not vanish simultaneously on $k_{1}+k_{2}$. This process can be continued by choosing the cubes in such an order that the distance of $k_{\varepsilon}$ from the origin is equal to or less than that for $k_{f}$ where $e<f$. Then the arbitrary $\beta$ 's for any cube can be extended as straight lines through the origin. Thus it is possible to find solutions of (1) which do not vanish simultaneously on $K$.

Since the matrix $C$ has maximum rank everywhere on $K$, this process can be continued until a $q \times q$ matrix

$$
D(u)=\left(\begin{array}{c}
\alpha_{i j}(u) \\
\beta_{k j}(u)
\end{array}\right)
$$

is obtained which is nonsingular everywhere on $K$. The matrix

$$
D(\phi(t))=\left(\begin{array}{c}
a_{i j}(t) \\
\beta_{k j}(\phi(t))
\end{array}\right)
$$

is nonsingular everywhere on $R$ and the functions $\beta_{k j}(\phi(t))$ are continuous on $R$. The functions $\beta_{k j}(\phi(t))$ can be approximated by polynomials $b_{k j}(t)$ so that the matrix $M$ is nonsingular everywhere on $R$.

NORTHWESTERN UNIVERSITY 